stools were managed conservatively and had no sequelae. Only one serious complication occurred in this group, when the battery lodged in a Meckel's diverticulum, causing liquefaction necrosis and perforation. This patient underwent laparotomy and survived.

On the basis of this information and our own experience we make the three following recommendations. Firstly, with few exceptions, ingestion of button batteries should be treated by observation alone. The obvious and important exception is when the battery is lodged in the oesophagus: this must always be treated as an emergency as oesophageal damage may occur within a few hours. Secondly, a chest radiograph should be obtained, especially if the battery is greater than $15 \mathrm{~mm}$ in diameter, as its size is the most important factor in determining whether it will pass beyond the oesophagus. Finally, if a battery that has gone beyond the oesophagus has split symptoms of mercury poisoning may occur and mercury concentrations should be measured.

1 Temple DM, McNeese MC. Hazards of battery ingestions. Pediatrics 1983;71:100-3.

2 Votteler TP, Nash JC, Rutledge JC. The hazards of ingested disc batteries in children. IAMA 1983;249:2504-6.

3 Litovitz TL. Button battery ingestions. JAMA 1983;249:2495-500.

(Accepted 8 May 1986)

Children's Hospital, Dublin 1

B KIELY, MRCPI, DCH, paediatric registrar

D GILL, FRCPI, DCH, professor of paediatrics

Correspondence to: Dr Kiely.

\section{An unusual case of Gardnerella vaginalis septicaemia}

Gardnerella vaginalis, first described in 1953 and originally thought to be a harmless commensal of the vaginal tract, has since been implicated as a cause of vaginitis, ${ }^{1}$ bacteraemias, ${ }^{23}$ and various genitourinary infections in men. ${ }^{+}$ Many bacteraemias caused by $G$ vaginalis in women, particularly post partum, have been reported. We report a case of $G$ vaginalis septicaemia with severe endotoxic shock in a previously fit man.

\section{Case report}

A 19 year old man was admitted to hospital with a 24 hour history of fever, rigors, pain in the loins, dysuria, and general malaise. Two hours before admission he had become progressively more drowsy and unresponsive. He had been circumcised as a child and had since suffered occasional episodes of dysuria and frequency associated with a meatal stricture. He was feverish $\left(41^{\circ} \mathrm{C}\right)$, had tachycardia ( 140 beats $/ \mathrm{min}$ ), and was hypotensive $(90 / 60 \mathrm{~mm} \mathrm{Hg})$. There were no focal neurological signs, and septicaemia with shock secondary to a urinary tract infection was provisionally diagnosed. He was admitted to the intensive care unit, resuscitated with intravenous fluids, and treated with cefuroxime and gentamicin. Over the next 24 hours he developed disseminated intravascular coagulation, which was treated with platelets, cryoprecipitate, and fresh frozen plasma, and acute renal failure, which was treated with dopamine and mannitol. Over the next 10 days his renal function and clotting factors returned to normal, and he made a full and unremarkable recovery. Subsequent investigation of the renal tract showed a small bladder with thickened walls and diverticulae.

Blood samples and a midstream specimen of urine were taken on admission before antibiotic treatment and dilatation of his meatal stricture. Microscopy of the urine showed numerous pus cells and Gram positive bacilli, but no organisms grew from urine samples incubated for 24 hours on cystine-lactose electrolyte deficient medium. After incubation for 48 hours on blood agar anaerobic cultures of both blood and urine yielded a heavy growth of a small poorly $\alpha$ haemolytic colony. Gram staining showed the colonies to be variable but made up predominantly of Gram positive bacilli similar to those seen on urine microscopy. The organism did not produce oxidase or catalase but fermented glucose, maltose, and mannite and hydrolysed hippurate. It was resistant to a disc containing $2.5 \mu \mathrm{g}$ metronidazole but was susceptible to a $50 \mu \mathrm{g}$ disc. It was also sensitive to penicillin, ampicillin, and cephalosporins and resistant to tetracycline and cotrimoxazole. Despite the poor haemolysis these characteristics were consistent with those of $G$ vaginalis. Indirect immunofluorescence of serum taken during the acute and convalescent phases showed an increase in antibody from a dilution of $<1: 8$ to $>1: 128$ against both the blood and urinary isolates.

\section{Comment}

There seems little doubt that this patient suffered a severe septicaemic illness caused by $G$ vaginalis in his urinary tract. It has been estimated that $G$ vaginalis is carried asymptomatically by $7-11 \%$ of men screened at a urology clinic. ${ }^{5}$ Several infections in men have implicated $G$ vaginalis, including balanoposthitis, urethritis, cystitis, and asymptomatic bacteriura. ${ }^{4}$ One case of bacteraemia also occurred after transurethral retropubic prostatectomy and was associated with fever, chills, and dysuria, and, though blood cultures were positive, urine culture was negative. ${ }^{3}$ The symptoms resembled those occurring in postpartum women rather than the severe endotoxic shock seen in our case. Carriage of the organism has been particularly associated with balanoposthitis, especially with a relative phimosis, and the meatal stricture seems to be the predisposing factor in this case. Although $G$ vaginalis is usually carried asymptomatically in the male urinary tract, it is important to recognise that it may occasionally cause a severe systemic illness.

We thank the department of microbiology, Sheffield University Medical School, for its help in confirming the identity of the organism.

1 Pheiffer TA, Forsyth MS, Dunfee MA, et al. Non specific vaginitis. Role of Haemophilus vaginalis and treatment with metronidazole. $N$ Engl 7 Med 1978;298:1429.

2 Reimer LG, Reller LB. Gardnerella vaginalis bacteraemia: a review of thirty cases. Obstet Gynecol 1984;64:170-2.

3 Patrick S, Garnett PA. Corynebacterium vaginale bacteraemia in a man. Lancet 1978;i:766.

4 Watson RA. Gardnerella vaginalis: genito urinary pathogen in men. Urology 1985;25:217-22.

5 Dawson SG, Ison CA, Csonka G, Easmon CSF. Male carriage of Gardnerella vaginalis. British Journal of Venereal Disease 1982;58:243-5.

\section{(Accepted 14 May 1986)}

Public Health Laboratory, Central Pathology Laboratory, Hartshill, Stoke on Trent ST4 7PX

J A WILSON, MB, DIPBACT, senior registrar

A J BARRATT, FIMLs, chief medical laboratory scientific officer

Correspondence to: Dr Wilson.

\section{Red cell morphology at high altitude}

In recent years the tourist industry has made areas at high altitude easily accessible. Exposure to high altitude may cause acute mountain sickness and its most severe complications-namely, high altitude pulmonary oedema and cerebral oedema.' The pathophysiology of acute mountain sickness is still unclear, but a contributing factor might be a change in the rheological properties of blood at high altitude. Recently, Rowles and Williams found an increased prevalence of abnormal red cell shapes, including echinocytes, in men who climbed above $4600 \mathrm{~m}$ for 39 days. ${ }^{2}$ Such abnormal shapes, especially echinocytes, can cause an increase in blood viscosity. ${ }^{3}$ No data are available on the influence of short term exposure to high altitude on the morphology of red cells; this prompted our study.

\section{Subjects, methods, and results}

We studied 10 healthy volunteers (five women, mean (SD) age $30 \cdot 4(6 \cdot 7)$ years, and five men, mean age $34 \cdot 4(5 \cdot 2)$ years). Blood $(100 \mu \mathrm{l})$ was drawn from an antecubital vein into a syringe containing $500 \mu \mathrm{l}$ of $1 \%$ glutaraldehyde. The first blood sample was taken at an altitude of $1190 \mathrm{~m}$ before the subjects ascended to $3400 \mathrm{~m}$ in a cablecar. After an overnight stay at $3600 \mathrm{~m}$ they reached an altitude of $4560 \mathrm{~m}$ (Cabin Marguerita, Italy) by foot the next day. Blood was drawn at that altitude after a six hour rest in the cabin, 24 hours after the first sampling. The red cells were fixed for 48 hours and then examined by light microscopy at a magnification of $\times 800$. They were classified blindly according to the classification of Bessis as discocyte; stomatocyte I (cup shaped (or convex-concave) red cell); stomatocyte II (red cell with a pronounced concavity); echinocyte I (irregularly contoured discocyte with up to five buckles); or echinocyte II (flat red cell with multiple spicules). ${ }^{+}$The data were analysed with Student's $t$ test for paired data.

The table shows the frequency distribution of the different types of cell. The discocyte is regarded as the equilibrium shape between two opposing transformations-that is, the stomatocytic and the echinocytic transformations. ${ }^{4}$ About $90 \%$ of the red cells were discocytes, and no difference was found in morphology at low and high altitudes.

Distribution of red cell shapes (\%) in 10 subjects at $1190 \mathrm{~m}$ and, 24 hours later, at $4560 \mathrm{~m}$ according to classification of Bessis. ${ }^{+}$Values are means $(S D)$

\begin{tabular}{|c|c|c|c|c|c|}
\hline \multirow[b]{2}{*}{ Altitude } & \multicolumn{2}{|c|}{ Stomatocytes } & \multirow[b]{2}{*}{ Discocytes } & \multicolumn{2}{|c|}{ Echinocytes } \\
\hline & II & I & & I & II \\
\hline $\begin{array}{l}1190 \mathrm{~m} \\
4560 \mathrm{~m}\end{array}$ & $\begin{array}{l}1.0(0.5) \\
1.0(0.6)\end{array}$ & $\begin{array}{r}11.9(4.3) \\
9.8(2.6)\end{array}$ & $\begin{array}{l}86 \cdot 9(4 \cdot 8) \\
89 \cdot 1(2 \cdot 4)\end{array}$ & $\begin{array}{l}0.3(0.4) \\
0.2(0.2)\end{array}$ & $\begin{array}{l}0.1(0 \cdot 2) \\
0\end{array}$ \\
\hline
\end{tabular}

